\title{
SIMULTANEOUS CONTROL OF PRODUCTION, REPAIR/REPLACEMENT AND PREVENTIVE MAINTENANCE OF DETERIORATING MANUFACTURING SYSTEMS
}

\author{
F. I. DEHAYEM NODEM ${ }^{\mathrm{a}}$, J. P. KENNE ${ }^{\mathrm{a}}$ and A. GHARBI ${ }^{\mathrm{b}}$ \\ École de technologie supérieure, 1100 Notre Dame Street West, Montreal, QC Canada H3C 1K3 \\ a Mechanical Engineering Department, Production Technologies Integration Laboratory \\ b Automated Production Engineering Department, Production System Design and Control Laboratory
}

\begin{abstract}
Abstrat
This paper presents a method to find the optimal production, repair/replacement and preventive maintenance policies for a degraded manufacturing system. The system is subject to random machine failures and repairs. The status of the system is considered to degrade with repair activities. When a failure occurs, the machine is either repaired or replaced. A replacement action renews the machine while a repair action brings it to a degraded operational state for which the next repair time increases as the number of repairs increases. A preventive maintenance action is considered to improve the reliability of the machine and therefore the disruptions caused by the machine failures are reduced. The decision variables are the production rate, the preventive maintenance rate and the repair/replacement switching policy upon machine failure. The objective of the study is to find the decision variables that minimize an overall cost, including repair, replacement, preventive maintenance, inventory holding and backlog costs over an infinite planning horizon. The proposed model is based on a semiMarkov decision process and the stochastic dynamic programming method is used to obtain the optimality conditions. A numerical example is given to illustrate the proposed model. A sensitivity analysis is considered to confirm the structure of the control policy and to illustrate the usefulness on the proposed approach.
\end{abstract}

Keywords: Manufacturing systems, numerical methods, optimal control, deteriorating systems, production, replacement/repair, preventive maintenance.

\section{Introduction}

In recent years there has been considerable interest in production planning and preventive maintenance control in manufacturing systems (see Dong-Ping (2009) and references therein). Such systems are subject to multiple uncertainties such as machines failures and repairs. Hence, repairable systems have attracted the attention of several researchers working in the field of deteriorating production systems as in Soro (2010). The repair costs may be very high after the machine experiences a large number of failures. In such a situation, it becomes more reasonable to replace the machine by a new one. A preventive maintenance action could also be considered in order to increase the operating time of the machine and reduce the disruptions of the production due to failures. 
In manufacturing environment, production activities, repairs, replacement and preventive maintenance actions are mutually interdependent. Gabriella et al. (2008) presented a general overview of models and discussed some sectors of interaction between maintenance and production. Until now, no work has been conducted which simultaneously provides the production planning, the repair/replacement and the preventive maintenance policies for systems that deteriorate with age and the number of failures. In this paper, we combine the production, repair/replacement and preventive maintenance control activities of a stochastic manufacturing system subject to random breakdowns. Many articles have been written on the interactions between production, corrective maintenance, preventive maintenance, replacement and machine deteriorations without integrating them in a unified model as in this paper.

The preventive maintenance is usually planned and aimed at increasing the reliability of a deteriorating system or decreasing the operating costs of repairable systems (see Boukas and Haurie (1990), Dellagi et al. (2007) and Yuan et al. (2001)). Boukas and Haurie (1990) included an agedependent machine failure rate and allowed the control to influence the jump rate from the machine operation mode to preventive maintenance mode. They determined production rate and maintenance rule which minimize the total expected cost of a two-machine system. However, with the numerical scheme adopted in their work, it remains computationally difficult to obtain the optimal control of a large scale FMS. To cope with this difficulty, Kenné and Boukas (2003) proposed a hierarchical control approach to determine the production and preventive maintenance policies for large scale manufacturing systems. A review of hierarchical approaches under uncertainties can be found in Sethi and Zhang (1994). Dehayem et al. (2009) used a hierarchical approach to develop a Semi-Markov Decision Model in order to make a lower level determination of the optimal repair and replacement policy for a system that deteriorates with age, and is subject to damage failures. Given that policy, at higher levels, they derived a production plan for the system over an infinite planning horizon. However, there is no evidence that the structure of the control policy obtained at lower level remains valid at the higher level of the hierarchy.

Yao et al. (2005) studied the joint preventive maintenance and production policies for an unreliable production system in which maintenance/repair times are non-negligible and random. Gharbi et al. (2007) assumed that failure frequencies can be reduced through preventive maintenance, and developed joint production and preventive maintenance policies depending on produced parts inventory levels. In their models, they assumed that maintenance actions always restore the machine to a perfect condition or 
does not change the failure rate of the system, such that the machine performs similarly from one failure to another. A more realistic assumption is that the machine does not always return to a perfect condition following a corrective maintenance. Such a repair is called an imperfect repair as in Liao (2007).

The imperfect repair model has been well documented, for repair and replacement policies in manufacturing systems (Kijima et al. (1988), Pérès and Noyes (2003)). Unfortunately, most available results concern only cases where production and demand satisfaction are not taken into account. The authors considered that repair, replacement and preventive maintenance take a small amount of time, and that they do not therefore significantly affect production activities (see Beichelt (1992), Makis and and Jardine (1993)).

If a machine is not initially new or is not new after each maintenance activity, it will clearly have different dynamics after each breakdown and repair. As stated by Pérès-Ocon and Torres-Castro (2002), under particular conditions involving exponential times, such a system can be modeled as continuoustime Markov chains. However, that is not the case when the probability distributions of operating and repair times follow general distributions. A semi-Markov process can then be used for modeling the system. Sloan (2008) developed a semi-Markov decision process model of a single-stage production system with multiple products and multiple maintenance actions. Such a model simultaneously determines maintenance and production schedules, while taking into account the fact that the equipment conditions affect the yield of each product differently. As can be seen from previous works, available studies did not simultaneously take into account the effect of a preventive maintenance control, repair/replacement, and the effect of increasing repair times with the number of failures.

Our aim in this paper is to develop optimal strategies for manufacturing systems, which take into account the deterioration of a machine in accordance with the number of failures (i.e., the repair time increases with the number of failures). Thus, repair activities depend on the machine's repair history and Markovian decision processes are no longer appropriate for the model of the control problem. The model proposed in this paper will simultaneously provide the following three policies:

- Repair/replacement switching policy based on the age of the machine and the number of failures above which the machine must be replaced if a failure occurs

- Preventive maintenance policy based on the age of the machine at which preventive maintenance must be performed

- $\quad$ Production policy based on the age of the machine and the stock level of finished goods 
The resulting simultaneous control approach consists in developing a Semi-Markov Decision Process (SMDP) in order to determine an optimal production, repair/replacement and preventive maintenance policies for the system. Such policies are determined in order to minimize inventory, backlog, repair, replacement and preventive maintenance costs over an infinite planning horizon.

The paper is organized as follows. In Section 2, we present the industrial context of the problem under study. In Section 3, we define the notations and assumptions used in the model. The problem statement is presented in Section 4. Numerical examples are presented in Section 5, and a sensitivity analysis is given in Section 6 to illustrate the usefulness of the proposed approach. Discussions and extensions of the proposed approach are given in Section 7 and the paper is finally concluded in Section 8.

\section{Industrial context}

The study presented in this paper has many applications, especially in production industry. As state by Badia and Berrade (2009), many engineering systems are subject to the so-called unrevealed failures. The unrevealed failures are constituted of those that are detected only by special tests, inspections or monitorings. Seal machines, filling machines, machining centers, grinders, milling, and many of tools machines are among examples. They have a large number of components (treadmill, ball screws, spindle heads, precision gear boxes, axis drive components, rotary tables, saddles, pallets and short). Those components stochastically deteriorate over time and hence the machine also deteriorates over time. Very often, parts of such machines are broken or damaged, although the machine is operational.

For example, worn nozzle, abrasives in pumped liquid, relief valve stuck, partially plugged or improperly adjusted, cavitations, worn bearing do not necessarily stop a liquid filling machine. A worn bearing will for example create knocking noise, while the machine still functioning. Thus, the failure of a machine is due to one or more removable and repairable components. Over time, the number of components to check and to repair at failure increases and thus the mean repair time increase also.

A preventive maintenance could serve to clean and adjust valves, control stroke and flow injection, replace worn nozzle with properly sized nozzle. Preventive interventions intend to reduce breakdowns risk and to maintain the smooth functioning of the machine.

The model presented in this paper is suitable for repairable manufacturing systems characterized by deterioration due to production and failures. The overall production system is renewed by a replacement after a sequence of successive failures and repairs; to be able to maintain a competitive advantage. 


\section{Notation and assumptions}

Throughout this article, we use the following notations and assumptions:

\subsection{Notations}

The following notations will be used to describe the proposed control model.

$x(\cdot) \quad$ stock level

$n(t) \quad$ number of failures at time $t$

$a(t) \quad$ age of the machine at time $t$

$\xi(\cdot) \quad$ stochastic process

d demand rate

$q_{\alpha \beta} \quad$ transition rate from mode $\alpha$ to $\beta$

$Q \quad$ transition rate matrix

$g(\cdot) \quad$ instantaneous cost

$c^{+} \quad$ holding cost per unit of item over per unit of time

$c^{-} \quad$ backlog cost per unit of item over per unit of time

$c_{0} \quad$ replacement cost

$c_{r} \quad$ repair cost

$J(\cdot) \quad$ total cost

$v(\cdot) \quad$ value function

$\rho \quad$ discount rate

$u(\cdot) \quad$ production rate of the manufacturing system

$\omega(\cdot) \quad$ preventive maintenance rate of the machine

$u_{\max } \quad$ maximal production rate of the manufacturing system

$\underline{\omega} \quad$ minimal preventive maintenance rate

$\bar{\omega} \quad$ maximal preventive maintenance rate

$s_{n}(\cdot) \quad$ age of the machine for a systematic replacement at failure

$N_{m} \quad$ Number of failure for a systematic replacement of the machine at failure

\subsection{Assumptions}

The mathematical model in this analysis has the following assumptions:

1) An infinite planning horizon. 
2) Failures of the machine are instantly detected and repaired (corrective maintenance).

3) The shortage cost depends on the shortage quantity and time (average value (\$/unit of time)).

4) The holding cost depends on the mean inventory level (average value (\$/unit of time)).

5) The cost of any replacement activity after failure is greater than the cost of a maintenance activity (corrective or preventive maintenance). In addition, the mean replacement time, (i.e., the mean time it takes to replace the machine after a failure) is lower than the time it takes to repair the machine when a failure occurs.

6) The mean time to repair increases with the number of failures

7) The mean replacement time is shorter than the mean time to repair for any number of failures

8) The mean time to replace the machine (i.e., the mean time the machine spends in operation before replacement at failure) is longer than the mean time to failure

9) The repair costs depend on the number of failures and are bounded non-decreasing functions

\section{Problem formulation}

In this section we describe a stochastic model for a deteriorating failure-prone machine producing one type of products to meet the demand of customers. The repairable machine goes through a finite number of successive failures before it is replaced by a new one. Therefore, we can assume that there exist a maximum number of failures $N$ that the machine can experience before replacement to guarantee the feasibility of the system. Thus, the number of failure $n(t) \in\{0,1, \cdots, N\}$. For a given number of failure, the inventory/backlog of the system, $x(t)$, evolves according to the following differential equation:

$$
\dot{x}(t)=u(t)-d, \quad x(0)=x
$$

where $x$ is the initial stock level and $u(t)$ is the production rate of the machine at time $t$; with $x(t)>0$ representing an inventory surplus and $x(t)<0$ a backlog of $-x(t)$. In the operational mode of the machine, we assume that $0 \leq u(t) \leq u_{\max }$ where $u_{\max }$ is its maximum production capacity. As the machine produces parts, its age increases. The machine age after the $n^{\text {th }}$ failure is given by:

$$
a_{n}(t)=a\left(t-t_{n}\right)+\theta_{n}, \quad t_{n} \leq t<t_{n+1}, t_{0}=0, \quad \theta_{0}=0
$$

where $t_{n}$ is the instant of the $n^{\text {th }}$ failure and $\theta_{n}$ is the virtual age of the machine after the $n^{\text {th }}$ repair given by the following expression:

$$
\theta_{n}=\psi \cdot a\left(t_{n}-t_{n-1}\right)+\theta_{n-1}, \quad n \geq 1
$$


where $\psi$ is known as repair intensity and is assumed to be constant in this paper $(0 \leq \psi<1)$. A repaired machine is as good as new if $\psi=0$; otherwise its virtual age increase with the number of failures (i.e., $\left.\theta_{0}<\theta_{1}<\theta_{2}<\cdots<\theta_{n}<\cdots\right)$. The function $a(t)$ is an increasing function of number of produced parts, since the last restart of the machine. It is described by the following differential equation:

$$
\dot{a}(t)=f(u(\cdot)), \quad a(T)=0
$$

where $T$ is the last restart time of the machine (i.e., $\left.T=0, t_{1}, t_{2}, \cdots\right)$ ).

The machine modes can be classified as operational, denoted by 1; under repair, denoted by 2; under replacement, denoted by 3 , and under preventive maintenance, denoted by 4 . The mode of the machine at time $t$ is then given by $\xi(t) \in \Omega=\{1,2,3,4\}$ such that:

$$
\xi(t)= \begin{cases}1 & \text { if the machine is operationnal } \\ 2 & \text { if the machine is under repair } \\ 3 & \text { if the machine is under replacement } \\ 4 & \text { if the machine is under preventive maintenance }\end{cases}
$$

The machine may randomly be at any of the four modes over an infinite horizon as described in appendix A where the transition diagram and details on transition rates are provided.

Let $s_{n}(x) \geq 0$ be the age at which if a failure occurs, the machine is replaced. The replacement switching age $s_{n}(x)$ is assumed to be a control variable for a given stock level $x$. Moreover, since each manufacturing system that deteriorates with age is to be replaced in the long term, we can assume that there exists an upper bound on the replacement age, $M_{u p}$, which is very large (compared to $S_{1}(\cdot)$ ) beyond which the machine is systematically replaced.

For any state variable $(x(t), \xi(t), a(t))$, we can identify the action to be undertaken at the $n^{\text {th }}$ failure as follow:

- If $n<N$ and $a(t)<s_{n}(x)$, then the machine is to be repaired

- Else the machine is replaced due to one of the following three situations:

a. The number of failures exceeds $N$

b. The age of the machine reaches $s_{n}(x)$

c. The age of the machine exceeds $M_{u p}$

The corrective maintenance of the machine is not perfect in the sense that the repair time increases with the number of failures. After corrective maintenance, the age is reset to the virtual age $\theta_{n}$. At the 
next failure, it will take much more time to repair the machine. Thus, repair activities depend on the machine's repair history and the system is thus described by a Semi-Markov Decision Process (SMDP). Hence, we shall refer to $Q(\cdot)$ as the transition matrix of the semi-Markov chain $\xi(\cdot)$. The control variables are the age $s_{n}(\cdot)$ after which the machine should be systematically replaced at the next failure, the production rate $u(\cdot)$ and the preventive maintenance rate $\omega(\cdot)$. Hence, the set of the feasible control policies $\Gamma(\alpha)$, including $s_{n}(\cdot), u(\cdot)$ and $\omega(\cdot)$, depends on the machine mode $\alpha \in \Omega$ and is given by:

$$
\Gamma(\alpha)=\left\{\left(s_{n}(\cdot), u(\cdot), \omega(\cdot)\right) \in \square^{3}, s_{n}(\cdot) \geq 0,0 \leq u(\cdot) \leq u_{\max } \text { Ind }\{\alpha=1\}, \underline{\omega} \leq \omega(\cdot) \leq \bar{\omega}\right\}
$$

The instantaneous cost associated to the considered planning problem is given by:

$$
g\left(\xi(t), a, x, n, s_{n}, u, \omega\right)=c^{+} x^{+}+c^{-} x^{-}+c_{r} \operatorname{Ind}\{\xi(t)=2\}+c_{0} \operatorname{Ind}\{\xi(t)=3\}+c_{m} \operatorname{Ind}\{\xi(t)=4\}
$$

where constants $c^{+}$and $c^{-}$are used to penalize inventory and backlog, respectively; $x^{+}=\max (0, x)$, $x^{-}=\max (0,-x), c_{r}$ is the repair cost, $c_{0}$ is a replacement cost, $c_{m}$ is the preventive maintenance cost; and the indicator function is defined as follows:

$$
\operatorname{Ind}(\Theta(\cdot))= \begin{cases}1 & \text { if } \Theta(\cdot) \text { is true } \\ 0 & \text { otherwise }\end{cases}
$$

The production planning problem considered in this paper is the determination of the optimal control policy $\left(s_{n}^{*}(\cdot), u^{*}(\cdot), \omega^{*}(\cdot)\right)$ minimizing the expected discounted cost given by:

$$
J\left(\alpha, a, x, n, s_{n}, u, \omega\right)=\mathrm{E}\left[\int_{0}^{\infty} \mathrm{e}^{-\rho t} g\left(\alpha, a, x, n, s_{n}, u, \omega\right) d t \mid x(0)=x, \xi(0)=\alpha, a(0)=a\right]
$$

where $\rho$ is a discount rate. The value function of the problem for a given number of failures $n$ is defined by:

$$
v(\alpha, a, x, n)=\inf _{\left(s_{n}, u, \omega\right) \in \Gamma(\alpha)} J\left(\alpha, a, x, n, s_{n}, u, \omega\right)
$$

The elementary properties of the value function $v(\cdot)$ given by equation (8) are presented in appendix B. The optimal control policy $\left(s_{n}^{*}(\cdot), u^{*}(\cdot), \omega^{*}(\cdot)\right)$ denotes a minimiser over $\Gamma(\alpha)$ of the right hand side of equation (B-1). This policy corresponds to the value function described by equation (8). Then, when the value function is available, an optimal control policy can be obtained as in equation (B-1). However, an analytical solution of equations (B-1) is almost impossible to obtain. The numerical solution of the HJB equations (B-1) is a challenge which was considered insurmountable in the past. Boukas and Haurie (1990) showed that implementing Kushner's method can solve such a problem in the context of production planning. The numerical methods used to solved the proposed optimality conditions are also presented in appendix B. In 
the next section, we provide a numerical example to illustrate the structure of the control policies.

\section{Numerical example}

This section provides a numerical example to demonstrate the obtained results. The purpose is to show the effectiveness of the close loop production, preventive maintenance and repair/replacement switching policies for a deteriorating system with a feedback on the number of failures, the age of the machine and the stock level. The instantaneous cost is the one described by equation (A-1), presented in Appendix A, with values of parameters given later in this section. In this example, the mean time to repair the machine increase with the number of failure due to the repair time given by equation (3) with $T_{21}(1)=1000$ and $r_{b}=0.5, d_{b}=-3$ (i.e., the mean time to repair the machine at the first failure is 1000 units of times and increase with the number of failures $n$ ). The transition rate is $q_{21}(n)=T_{21}^{-1}(n)$ with $q_{21}(1)=0.001$.

The failure rate and the corresponding operational time is obtained from a Weibull distribution with scale parameter $\lambda=0.05$ and a shape parameter $\alpha=3$. In addition, $u_{\max }=0.4, d=0.25, q_{31}=10$, $q_{41}=0.25, \psi=0.01$. The other parameters are presented in table 1 .

\begin{tabular}{|c|c|c|c|c|c|c|c|c|c|}
\hline$c_{0}$ & $c_{r}$ & $c_{m}$ & $h_{x}$ & $h_{a}$ & $c^{+}$ & $c^{-}$ & $\rho$ & $\underline{\omega}$ & $\bar{\omega}$ \\
\hline 20000 & 100 & 10 & 0.5 & 1 & 2 & 150 & 0.05 & 0.0001 & 0.1 \\
\hline
\end{tabular}

Table 1. Parameters of the numerical example

The computational domain $D$ is defined by:

$$
D=\{(x, a, n):-5 \leq a \leq 15,0 \leq a \leq 100,0 \leq n \leq 20\}
$$

The policy improvement technique is used to solve the system of four equations obtained from (B-1 for each mode of the machine. The obtained results are presented in this section to clearly illustrate the production, preventive maintenance and repair/replacement switching policies.

\section{a) Production policy}

The optimal production policy $u^{*}(a, x, \cdot)$, illustrated by the figure $1(\mathrm{a})$, indicates the production rate, for a given number of failures $n$, for each stock level $x(t)$, and each age $a(t)$ of the machine, 


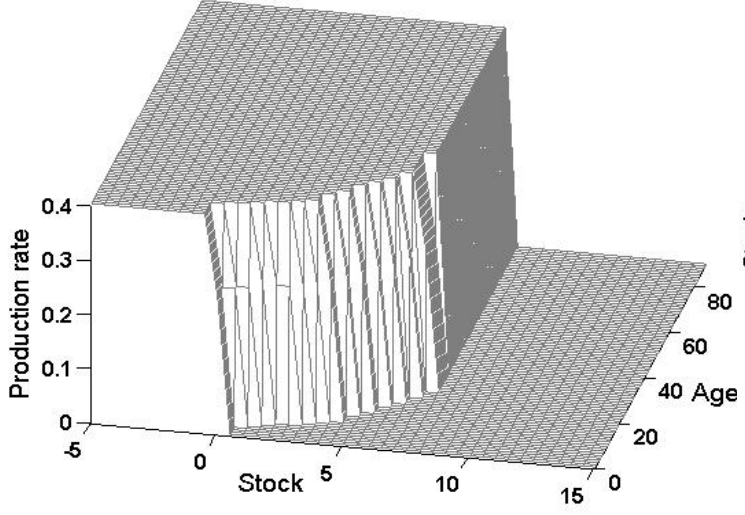

(a): $u^{*}(x, a, \cdot)$

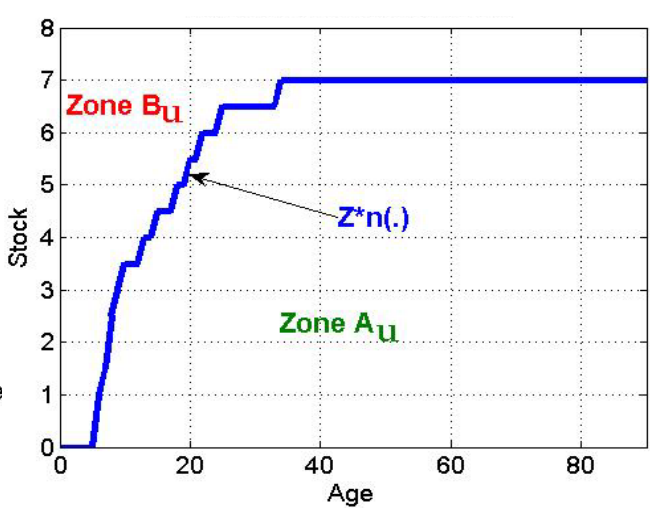

(b): $z_{n}^{*}(\cdot)$

Figure 1. Optimal production rate and its boundary

To illustrate the optimal production policy, we use its boundary given by figure 1(b). The boundary of the optimal production policy is the optimal stock level $z_{n}^{*}(\cdot)$ such that, if the stock level in the system $x($.$) is less than the optimal stock level, the production should be at a maximum rate to reach the optimal$ stock level. Once the stock level in the system is equal to the optimal stock level, the production should be at the demand rate. If the stock level in the system exceeds the optimal stock level, then we do not produce. The optimal production policy recommends a production in zone $\mathrm{A}_{\mathrm{u}}$ to reach the stock level $z_{n}^{*}(\cdot)$ and no production in zone $\mathrm{B}_{\mathrm{u}}$.

The optimal stock level is given by figure 2(a) for $n=1,2,6,9$ and by figure 2(b) for $n>9$.

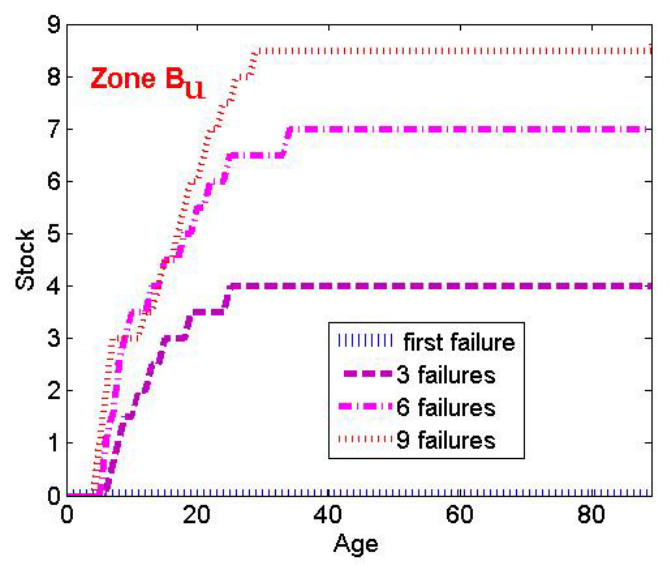

(a): Low number of failures

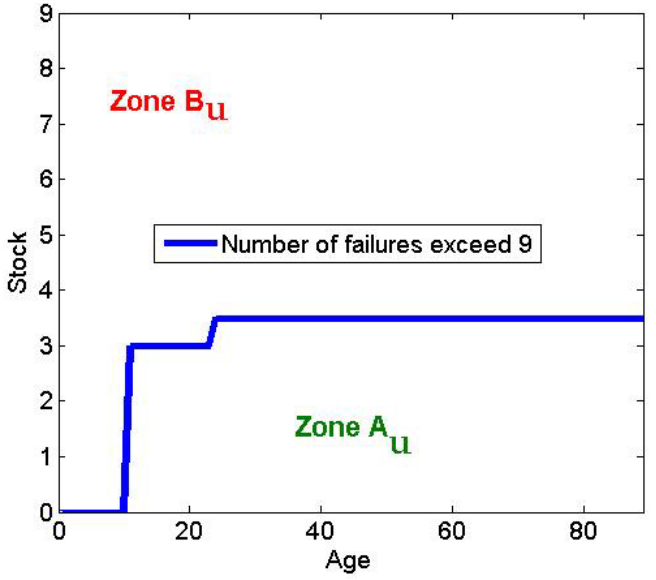

(b): High number of failures 
Figure 2. Optimal stock levels $z_{n}^{*}(\cdot)$

As in figure 2(a), when the machine has not yet had its first failure, while awaiting the first failure, the number of parts to hold in inventory is equal to zero. The corresponding curve matches with the line $x=0$ and the zone $A_{u}$ is inexistent. When the machine has experienced its $n^{\text {th }}$ failure, the number of parts to hold in inventory increases and attains a maximal value at $\max \left(z_{n}^{*}(\cdot)\right)$. This maximal number of parts to hold in inventory increases with the number of failures until a given maximal number of failures, as can be seen in figures 2(a) and 2(b). The corresponding maximal number of failures in this example is 9, as shown in figure 2(b). Moreover, when the number of failures exceeds 9, the zone $A_{u}$ and zone $\mathrm{B}_{\mathrm{u}}$ are constant.

As illustrated by figure 3, the production policy is characterized by three parameters $A_{1}(n), A_{2}(n), z_{m}(n)$ such that:

- $\quad$ if the age of the machine $a(t)$ is less than $A_{1}(n)$, the number of parts to hold in inventory is zero (i.e., $\left.z_{n}^{*}(\cdot)=0\right)$.

- If the machine's age $a(t)$ is such that $A_{1}(n) \leq a(t) \leq A_{2}(n)$, then the number of parts to hold in inventory must be brought from zero to $z_{m}(n)$.

- $\quad$ If the machine’s age reaches $A_{2}(n)$, the stock must be maintained at level $z_{m}(n)$.

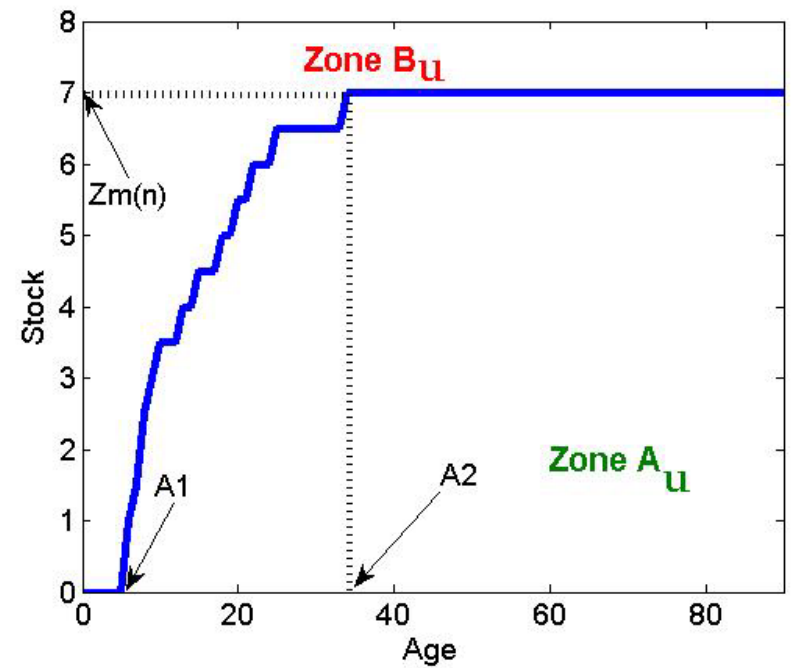

Figure 3. Production policy $\mathrm{z}_{n}^{*}(\cdot)$ parameters 
Figure 4 gives the production policy for several failure numbers. The number of parts to hold in inventory increases as the age $a(t)$ and the number of failures $n$ increase.

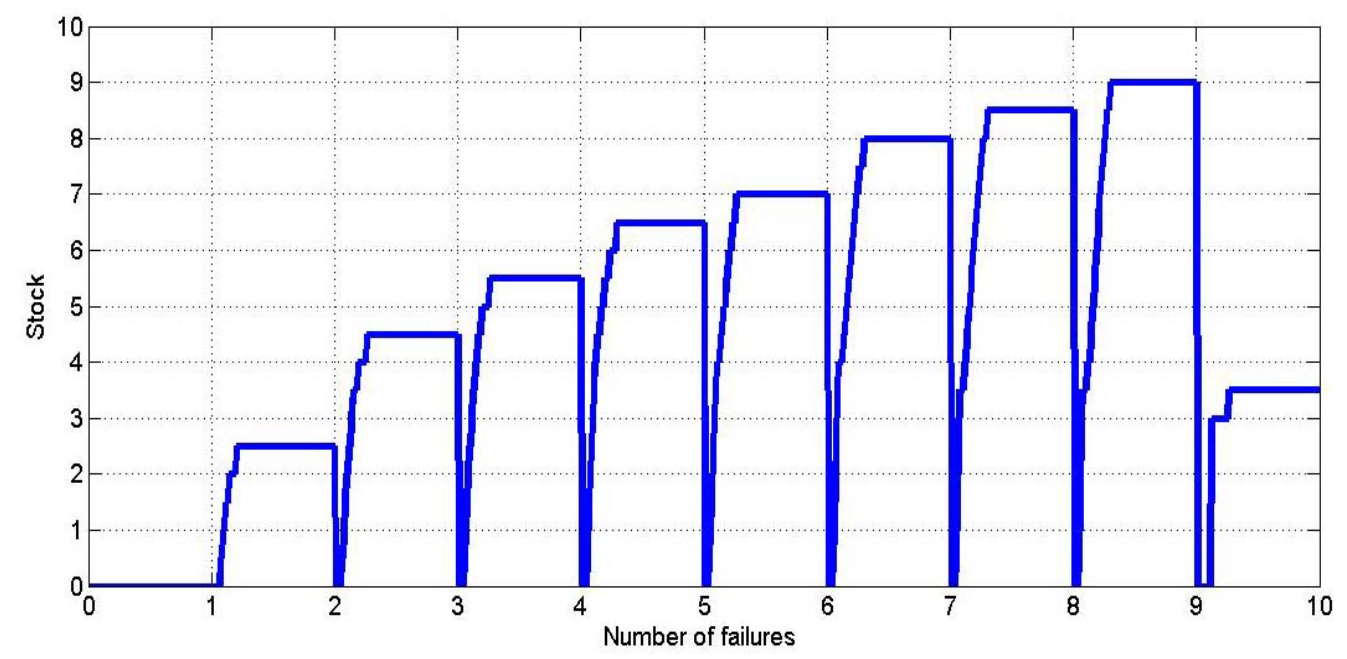

Figure 4. Number of parts to hold in inventory for each number of failures

The maximal number of parts to hold in inventory for several numbers of failures presented in figure 5 increases from one failure to another. This is logical because if the machine is to be repaired, it will take a greater amount of time. The optimal policy recommends holding a small number of parts in inventory after failure 9 because the machine will be renewed when failure 10 occurs.

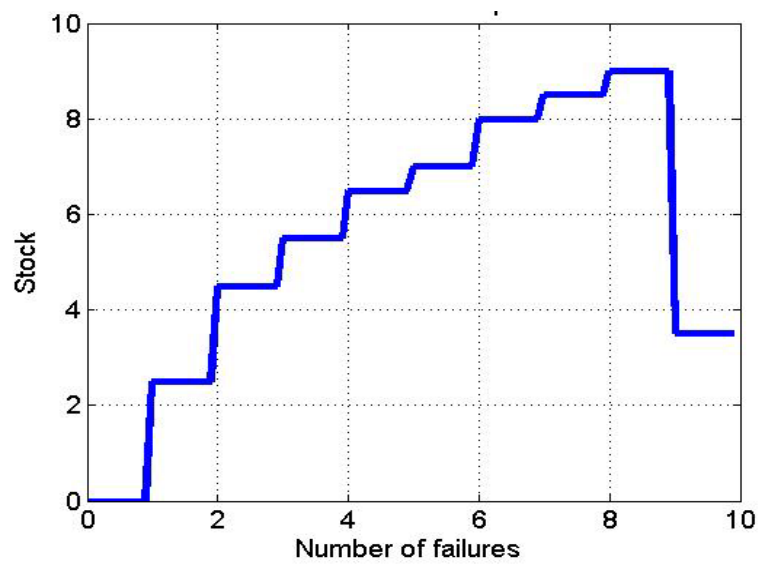

Figure 5. Maximal number of parts $z_{m}(\cdot)$

Based on the results from figures 1 to 5 , the production rate is given by the following machine failure dependent hedging point: 


$$
u^{*}(1, a, x, n)= \begin{cases}u_{\max } & \text { if } x(t)<z_{n}^{*}(\cdot) \\ d & \text { if } x(t)<z_{n}^{*}(\cdot) \\ 0 & \text { otherwise }\end{cases}
$$

with $z_{n}^{*}(\cdot)= \begin{cases}0 & \text { if } a(t) \leq A_{1}(n) \\ \in] 0, z_{m}(n)[ & \text { if } A_{1}(n)<a(t)<A_{2}(n) \\ z_{m}(n), & \text { if } a(t) \geq A_{2}(n)\end{cases}$

It is important to note from equation (13) that when $a(t)>A_{1}(n)$, the stock level should be brought from 0 in order to reach $z_{m}(n)$ at $a(t)=A_{2}(n)$. The stock level is maintained at the value $z_{m}(n)$ when $a(t) \geq A_{2}(n)$.

\section{b) Preventive maintenance policy}

The obtained optimal preventive maintenance policy is presented in figures 6(a), 6(b) and 6(c

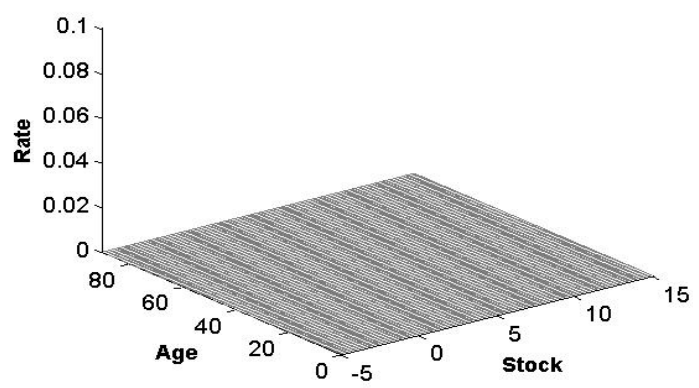

(a): Before 1 failure

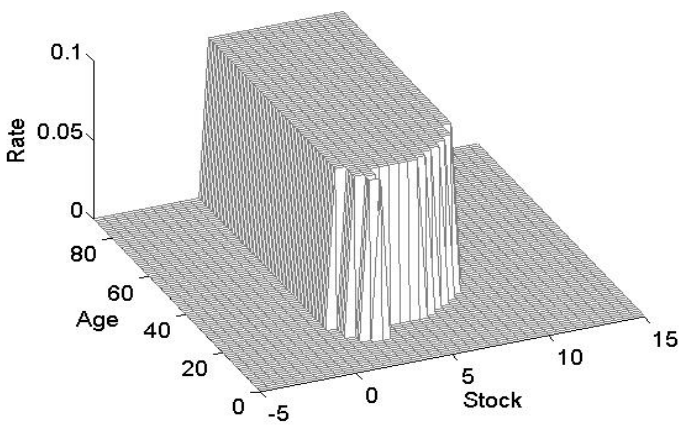

(b): Before 4 failures

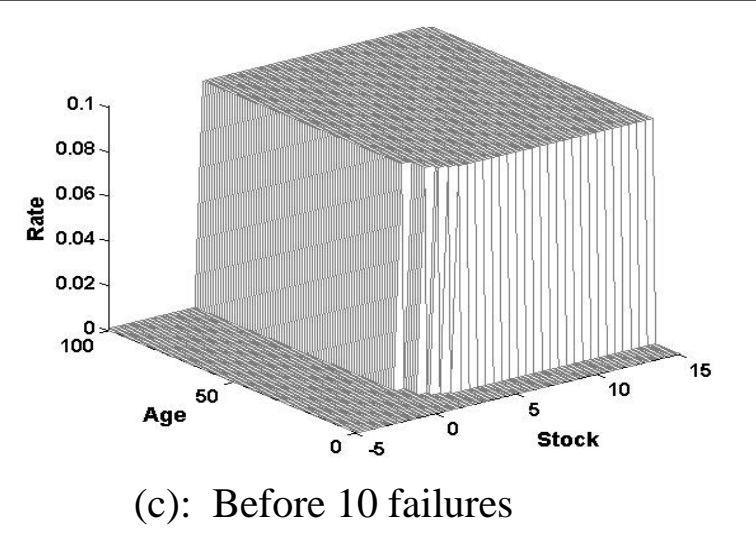

Figure 6. Preventive maintenance policy $\omega^{*}(\cdot)$

). It shows that when the machine has not yet experienced its first failure (figure 6(a)), a preventive maintenance action is not recommended. Conversely, when the number of failures increases (figures 
6(b) and 6(c)), machine age dependent preventive maintenance actions are recommended. However, it depends on the age of the machine. Throughout the rest of the paper, we use the boundary of the optimal preventive maintenance, namely Trace of PM, present in figure 7 to analyze the evolution of the optimal preventive maintenance policy. The boundary $T_{\omega}(x, n)$ of the optimal preventive maintenance policy divided the plan $(x, a)$ in two zones:

- In zone $\mathrm{A}_{\omega}$, it is recommended to preventively send the machine to maintenance.

- In zone $\mathrm{B}_{\omega}$, the optimal preventive maintenance policy consists of not proceeding to a preventive maintenance of the machine.

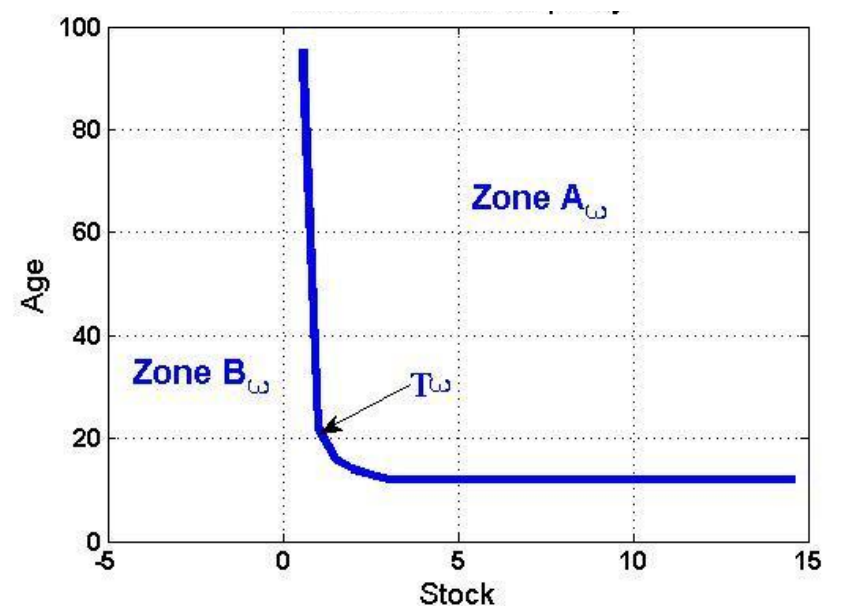

Figure 7 . Trace of the preventive maintenance policy $T_{\omega}($.

The Trace of PM is presented in figure 8 for different number of failures.

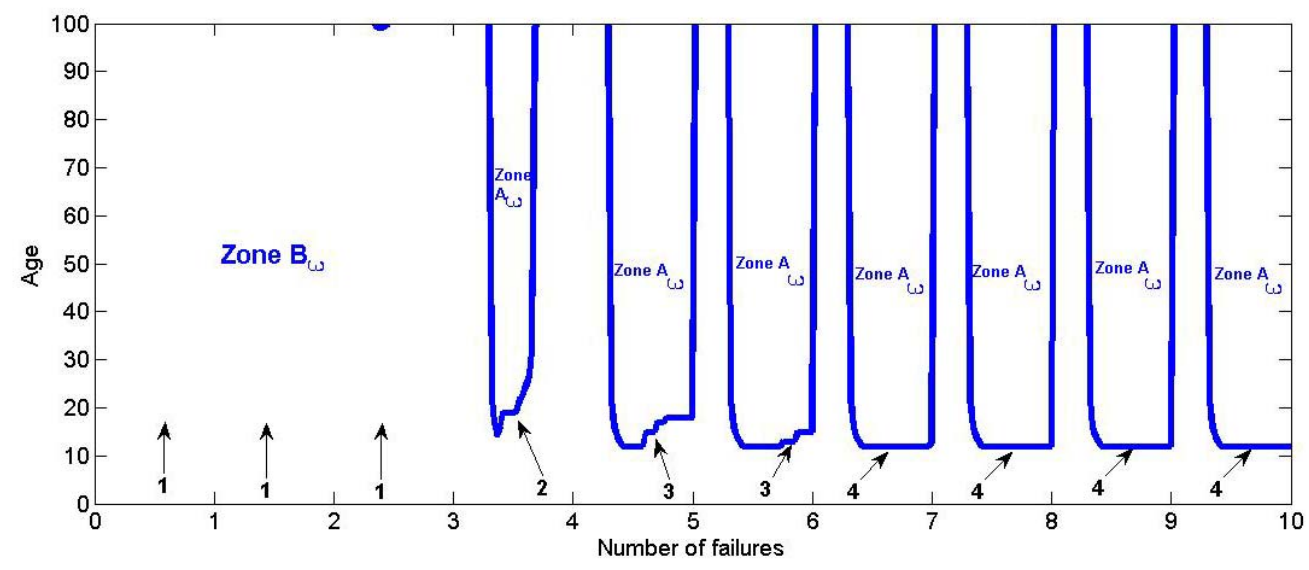

Figure 8. Boundary of the preventive maintenance policy for several failures 
When the machine has experienced less than 3 failures (indicated by arrow 1 in figure 8 ), the zone $A_{\omega}$ is non-existent. There is no preventive maintenance action before the $3^{\text {th }}$ failure. The zone $\mathrm{A}_{\omega}$ appears between failure 3 and failure 4, as indicated by arrow 2 in figure 8 . It then increases, as indicated by arrow 3, and attains a constant value indicated by arrow 4, from failure 6 to failure 9 .

The preventive maintenance actions are triggered according to a machine age dependent policy described in figures 6, 7 and 8. Such a policy states that preventive maintenance should be performed at rate $\omega^{*}(\cdot)$ given by the following equation:

$$
\omega^{*}(1, a, x, n)= \begin{cases}\underline{\omega} & \text { if } a(t)<T_{\omega}(\cdot) ; \text { i.e., the zone } \mathrm{B}_{\omega} \text { is active } \\ \bar{\omega} & \text { otherwise; i.e., the zone } \mathrm{A}_{\omega} \text { is active }\end{cases}
$$

where $T_{\omega}(\cdot)$ is the age limit for preventive maintenance before the $n^{\text {th }}$ failure of the machine.

\section{c) Repair/replacement switching policy}

The obtained repair/replacement switching policy $s_{n}^{*}(x)$ is presented in figures 9(a) and 9(b).

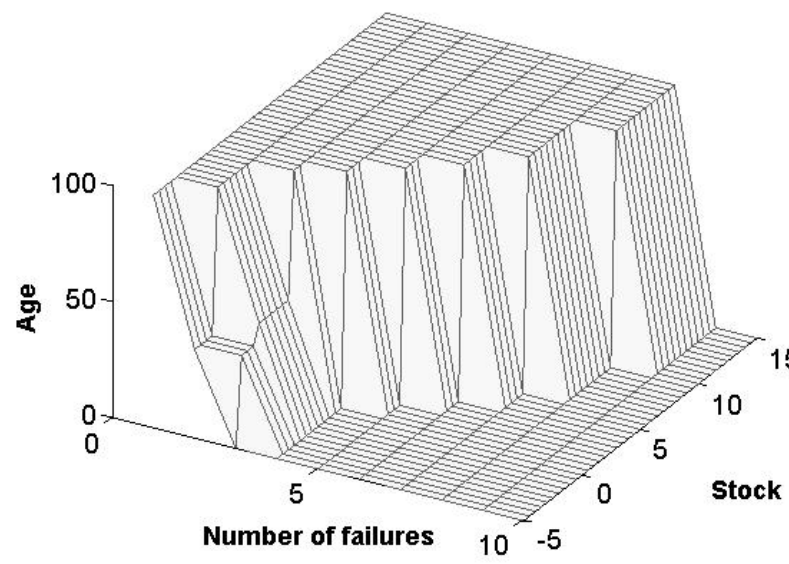

(a): $s_{n}^{*}(\cdot)$

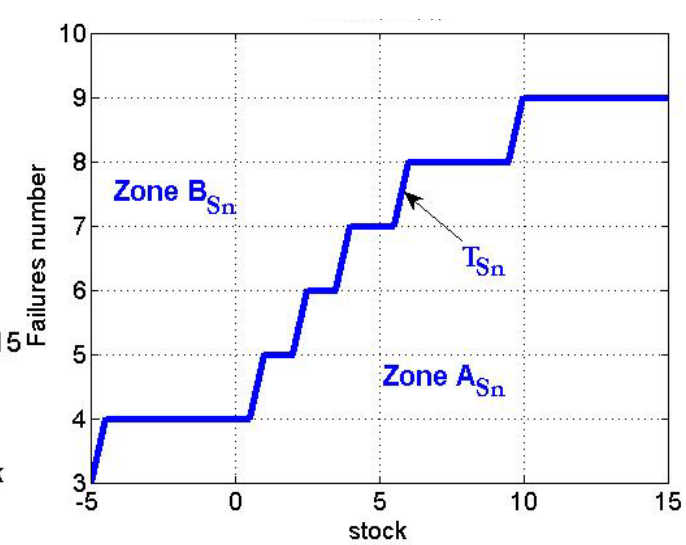

(b): $T_{s n}$

Figure 9. Repair/replacement policy and its boundary

From figure 9(a), the age $s_{n}^{*}(x)$ over which the machine should be replaced if a failure occurs, decreases with the number of failures and increases with the stock level. The age $s_{n}^{*}(x)$ is equal to 0 when the machine has experienced 9 failures. It means that while awaiting the $10^{\text {th }}$ failure, the replacement age is 0 , no matter the stock level. The machine will experience a maximum number of 9 
failures before systematic replacement at the next failure, no matter its age and the stock level. Hence, $N_{m}^{*}=9$.

The boundary of the repair/replacement switching policy, denoted by $T_{s n}$ is presented in figure 9(b). Such a boundary divides the plan $(x, n)$ in two zones:

- In zone $B_{S n}$, the machine is systematically replaced if a failure occurs.

- In zone $\mathrm{A}_{S n}$, the machine is repaired if a failure occurs above the replacement age $s_{n}^{*}(x)$.

Let $R_{n}(a, x)$ denotes a function with value 1 if a repair action is undertaken after the $n^{\text {th }}$ failure; and 0 if not. Based on the above results and with such a notation, the repair/replacement switching policy is given by the following expression:

$$
R_{n}(a, x)=\left\{\begin{array}{l}
1 \text { if } a(t)<s_{n}^{*}(x) \text {; i.e., the zone } \mathrm{A}_{S n} \text { is active } \\
0 \text { otherwise; i.e., the zone } \mathrm{B}_{S n} \text { is active }
\end{array}\right.
$$

with $s_{n}^{*}(x)$ given in figure 9(a).

In the next section, we will confirm the structure of the obtained control policies through a sensitivity analysis and illustrate the usefulness of the proposed approach.

\section{Sensitivity and Result Analysis}

A sensitivity analysis is presented in this section on cost parameters to confirm the structure of the obtained control policies given by equations (13)-(15) and to illustrate the contribution of this paper. The sensitivity of the control policies are analyzed according to the variation of the cost of repair, the cost of replacement and the cost of preventive maintenance.

\section{a) Variation of the repair cost}

Figure 10(a) illustrates the maximal number of parts to hold in inventory for four replacement cost values $c_{r}=5,10,20$ and 30 . The results show that the variations of the repair cost have no significant effect on the threshold value $z_{m}(\cdot)$ but they affect the repair/replacement switching policy as shown in figure 10(b). From figure 10(b), when the repair cost varies and takes the values 5, 10, 20 and 30, the machine is systematically replaced after the $13^{\text {th }}, 10^{\text {th }}, 7^{\text {th }}$ and $6^{\text {th }}$ failures, respectively. The trend is then to replace the machine early when the repair cost increases. Thus, if the machine is to be replaced at the next failure, the number of parts to hold in inventory while awaiting that failure is not high. 
The preventive maintenance policy, shown in figure 10(c), is significantly affected by the variation of the repair cost. The area $A_{\omega}$ increases with the repair cost, that is, as repair costs increase, the preventive maintenance is recommended more often. It is clear from the above results that the repair costs significantly influence the obtained optimal control policies. The machine is replaced earlier (it experiences a lower number of failures) when the repair cost increases, and the optimal threshold depends on the number of failures the machine experiences and the preventive maintenance area increases.

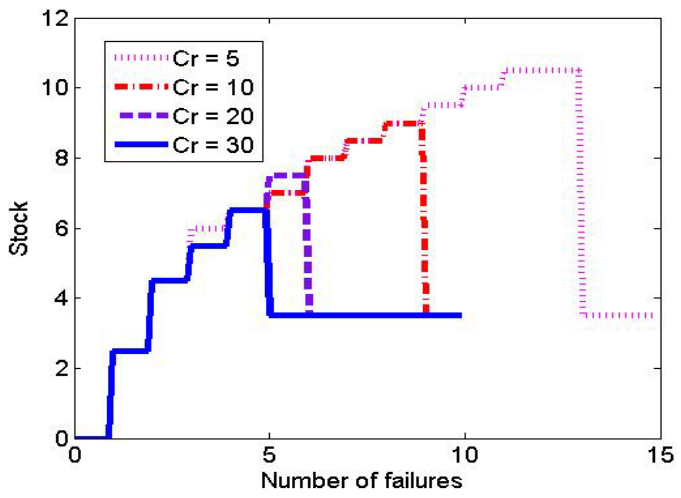

(a): $z_{m}(n)$

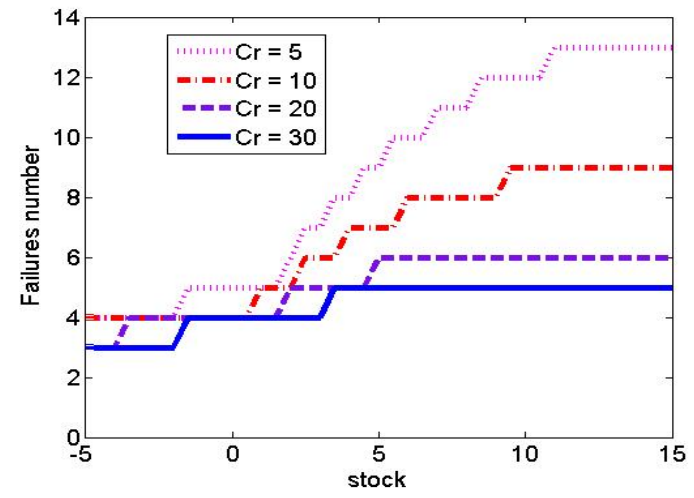

(b): Boundary of $s_{n}(\cdot)$

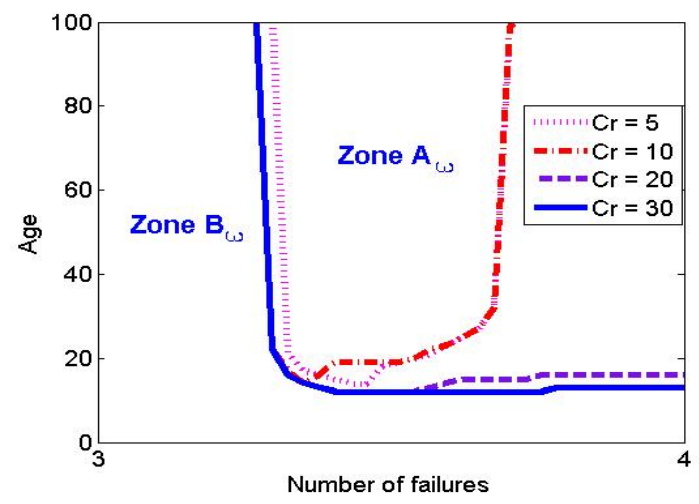

(c): $T_{\omega}(\cdot)$ for a given failure number $n$

Figure 10. Variation of the repair cost

\section{b) Variation of the replacement cost}

The variation of the replacement cost $c_{0}$ affects the optimal threshold stock level as shown in figure 11(a). After a certain number of failures, the threshold level is significantly different from one replacement cost to another. For a replacement cost value of 5,000, from the $5^{\text {th }}$ failure, there is no need to stock parts, while for a replacement cost value of 20,000, products should be stocked up to 9 failures. 
Further, when the replacement cost is 5,000, as illustrated in figure 11(b), the machine is systematically replaced at the $6^{\text {th }}$ failure. From figure $11(\mathrm{c})$, we notice that there are only three curves instead of four curves given that the curve representing the value $c_{0}=5,000$ is not illustrated. This is because no preventive maintenance is recommended when replacement cost is 5,000. This observation is logical in that, since the replacement is not expensive, it is better to replace the machine with a new one as compared to sending it to preventive maintenance and then expecting another failure.

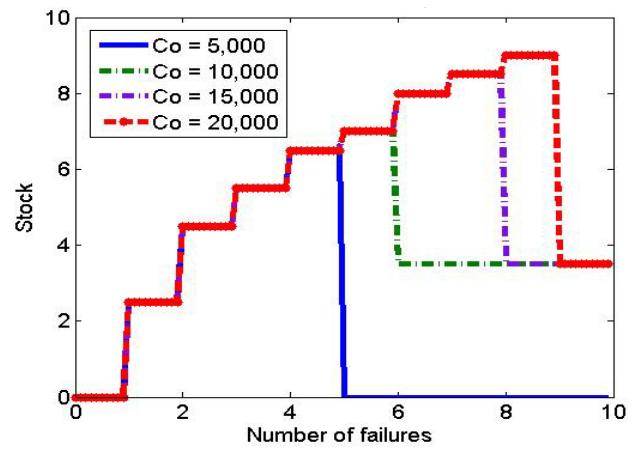

(a): $z_{m}(n)$

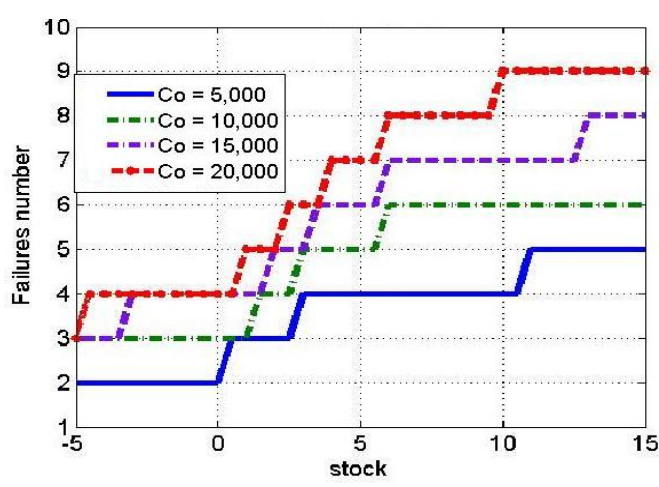

(b): Boundary of $s_{n}^{*}(\cdot)$

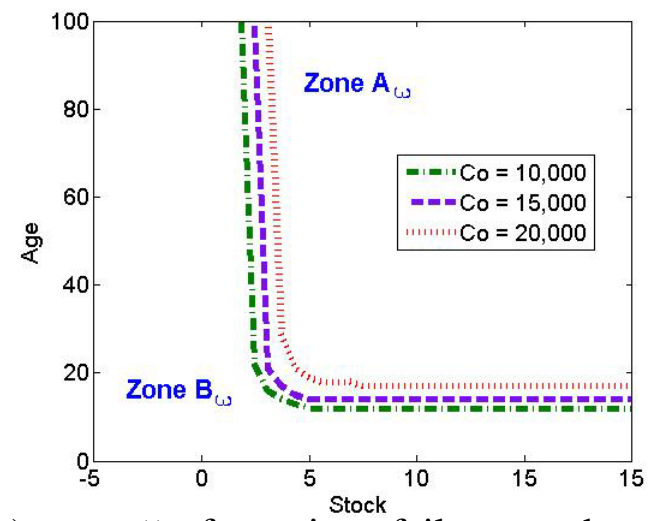

(c): $\quad T_{\omega}(\cdot)$ for a given failure number $n$

Figure 11. Variation of the replacement cost

\section{c) Variation of the preventive maintenance cost}

The variation of the preventive maintenance cost $c_{m}$ has a relatively small effect on the production and repair/replacement policies, as shown in figures 12(a) and 12(b). On the other hand, it significantly affects the preventive maintenance policy, as illustrated in figure 12(c), where we observe that for a high preventive maintenance cost value $\left(c_{m}=300\right)$, the area where a preventive maintenance is recommended is reduced. 


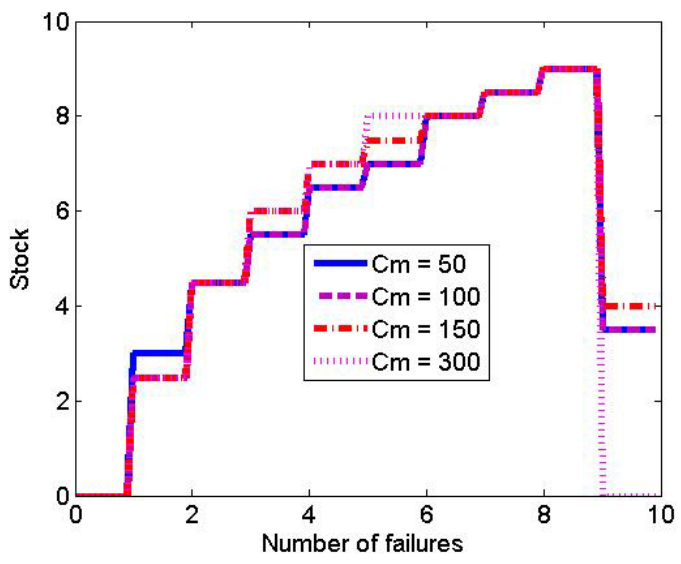

(a): $z_{m}(n)$

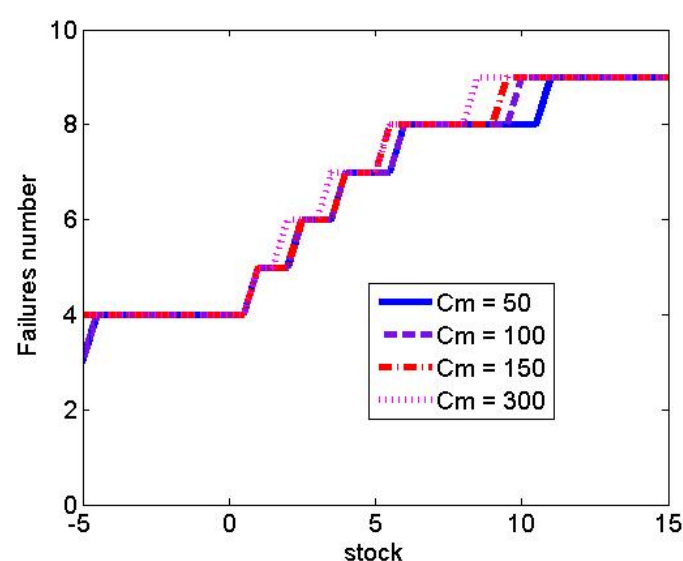

(b): $s_{n}^{*}(\cdot)$

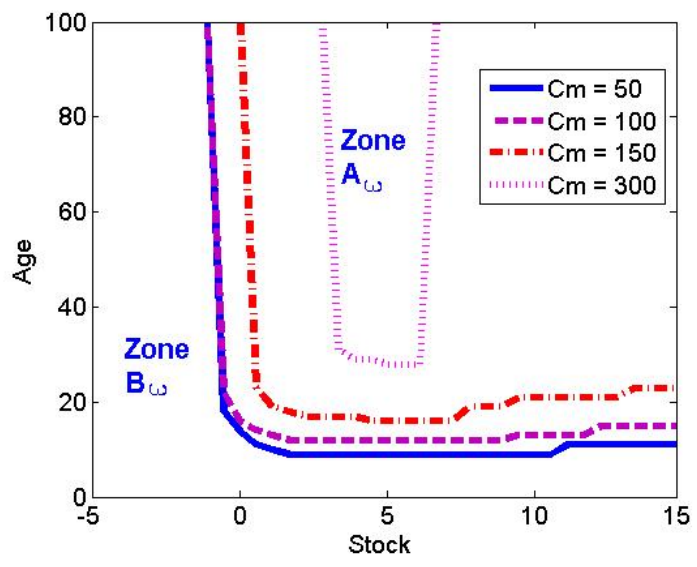

(c): $T_{\omega}(\cdot)$ for a given failure number $n$

Figure 12. Variation of the preventive maintenance cost

These conclusions on the influence of preventive maintenance cost on production and preventive maintenance policy are close to those obtained by Gharbi et al. (2007). They observed, when formulating an analytical model for the joint determination of an optimal age-dependent buffer inventory and preventive maintenance policy in a production environment, that increasing preventive maintenance costs reduces preventive maintenance frequency and have no significant effect on the trend of the production policy as in this paper. 


\section{Discussions and extensions}

It is important to note that in many situations, the dynamics of several variables change after breakdowns as a result of the machine degradation phenomenon. As shown in the above results, taking into account some degradation with age after a machine failure leads to a policy comprising several critical threshold values, which increase from one breakdown to another. The results obtained indicate that the optimal production, repair/replacement and preventive maintenance policies for the considered manufacturing system are characterized by a special structure. Such a structure is defined by:

- three parameters for production (i.e., $z_{m}(n), A_{1}(n), A_{2}(n)$ ),

- two parameters for the repair/replacement switching policy (i.e. $\left.s_{n}(x), N_{m}\right)$

- one parameter for the preventive maintenance policy (i.e. $\left.T_{\omega}(\cdot)\right)$.

The overall control policy given by equations (13), (14) and (15) is completely defined by the values of six parameters $\left.z_{m}(n), A_{1}(n), A_{2}(n)\right), s_{n}(x), N_{m}$ and $T_{\omega}(\cdot)$ for production, repair/replacement switching and preventive maintenance policies.

For a given stock level, the structure of the optimal repair/replacement policy is an extension of the one obtained by Makis and Jardine (1993) and Love et al. (2000). As shown in section 5, their results appear as a particular case of the repair/replacement switching policy presented in this paper. Our results state that if at the $n^{\text {th }}$ failure, the age of the machine is above a certain critical value, the machine should be replaced. Otherwise, an imperfect repair should be undertaken. The repair/replacement policy present in this work is more realistic and appropriate for manufacturing systems because it takes into account the stock level in the system, the rate at which parts should be produced and a preventive maintenance policy.

Through the observations from the sensitivity analysis, it clearly appears that the proposed approach, based on a simultaneous control of production, repair/replacement and preventive maintenance strategies, provides more realistic results in the context of stochastic manufacturing systems. For a more complex manufacturing system consisting of $m$ machines, producing $k$ different part types, the control policy depends on $6 \times m \times k$ parameters The solution of HJB equations (as in equation (12)) is almost impossible for large $m$ and $k$ since the dimension of the numerical scheme to be implemented increase exponentially with the complexity of the system. For the aforementioned problem (i.e., with $m$ and $k$ ), a combination of the control theory and the simulation based experimental design as in Gharbi and Kenné (2003) can be used to obtain a near optimal control policy. This could give the possibility to develop a more general model in 
production planning including capacity limits, multi-product management and uncertainties on product demands.

\section{Conclusion}

In this paper, a production, repair/replacement and preventive maintenance planning problem in manufacturing systems has been studied. The objective of the study was to determine how to produce while the machine is in operation, when to replace versus repair the machine if a failure occurs and when to perform a preventive maintenance, if any, in order to minimize the overall incurred cost. Our formulation included mode- and control-dependent jump rates, and allows us to represent the increasing probability of failures due to machine aging and increasing repair time resulting from imperfect repair activities. We used reset functions, which permit repair and preventive maintenance activities to restore the machine's age to level 0 , and replacement, which provides a new machine. Thus, we extended the repair and replacement switching age control model to the case where production and preventive maintenance rates are controlled. Due to the fact that the repair activities of the machine depend on the repair history, we proposed to use a semi-Markov decision process to determine the optimal policies. We provide a numerical example, and through a couple of sensitivity analyses, we showed that the structure of the results obtained is maintained. 


\section{Appendix A.}

The diagram in figure A-1 illustrates the transitions between different modes of the machine.

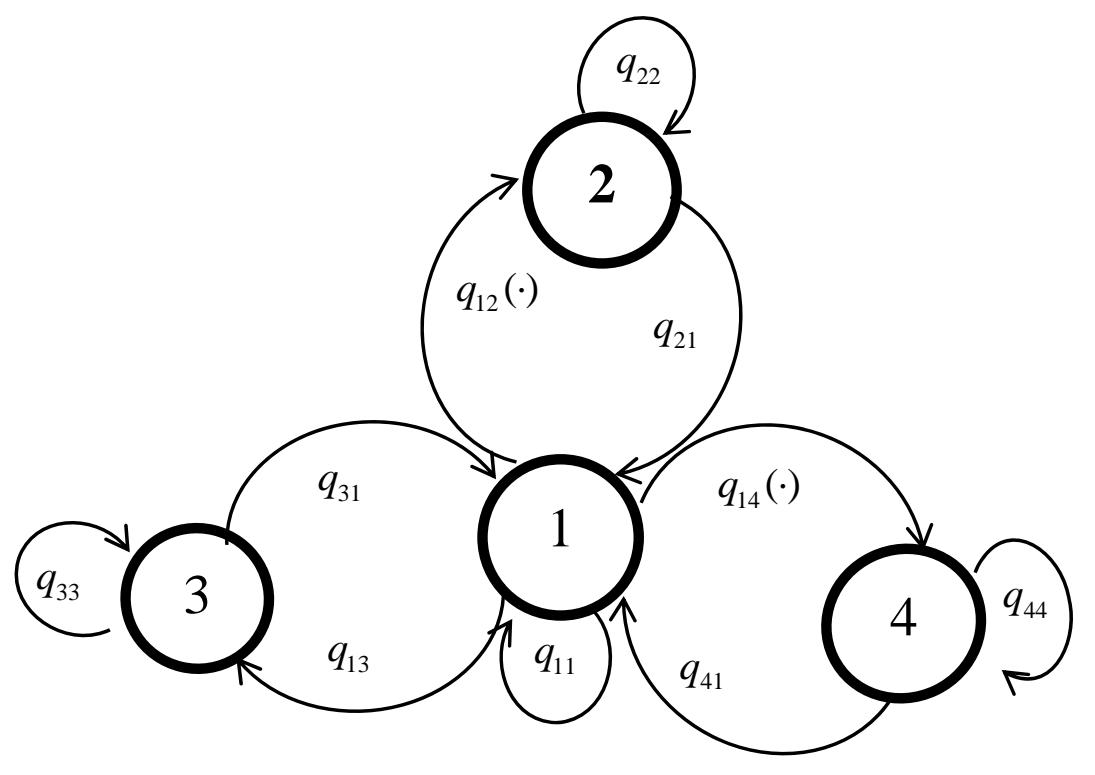

Figure A-1. State transition diagram

The transition rates $q_{\alpha \beta}, \alpha, \beta \in \Omega$, are described as follow:

- The failure rate $q_{12}\left(a_{n}(t)\right)$ and the replacement rate $q_{13}\left(a_{n}(t)\right)$ of the machine depend on its age $a(t)$ and are obtained from a machine age dependent Weibull distribution.

- The machine is replaced at a constant rate $q_{31}$.

- The repair rate $q_{21}(n)$ is the inverse of $T_{21}(n)$ To define $T_{21}(n)$, let $t_{21}(n)$ be the repair time after the $n^{\text {th }}$ failure, and $T_{21}(n)$ the expectation of $t_{21}(n)$. The sequence $\left\{t_{21}(n), n=1,2, \cdots, N\right\}$ forms a non-decreasing arithmetic-geometric process with parameters $d_{b} \leq 0$ and $0<r_{b} \leq 1$. We have for example $\mathrm{E}\left(t_{21}(1)\right)=T_{21}(1) \geq 0$ and $T_{21}(1)=0$ means that the repair time is negligible. As in Leung (2006), the non-decreasing repair time $T_{21}(n)$ is given by:

$$
T_{21}(n)=\frac{T_{21}(1)}{r_{b}^{n-1}}-(n-1) d_{b}, \quad n=1,2, \cdots, N
$$

- The transition rate from operational mode 1 to preventive maintenance mode 4 (i.e., $q_{14}=\omega(\cdot)$ ) is a control variable. The inverse of $\omega(\cdot)$ represents the expected delay between the decision to trigger preventive maintenance actions and the effective switch from operational to preventive 
maintenance mode (see Boukas and Haurie (1990) for details). The following constraints hold for the preventive maintenance jump rate:

$$
\underline{\omega} \leq \omega(\cdot) \leq \bar{\omega}
$$

where $\underline{\omega}$ and $\bar{\omega}$ are the minimal and maximal preventive maintenance rates of the machine.

- The machine mode changes from preventive maintenance to operational mode at a constant rate $\mathrm{q}_{41}$.

Let $Q(\cdot)=\left(q_{\alpha \beta}(\cdot)\right)$ denotes a $4 \times 4$ matrix such that:

$$
\begin{aligned}
& q_{\alpha \beta}(\cdot) \geq 0, \quad \alpha \neq \beta \\
& q_{\alpha \alpha}(\cdot)=-\sum_{\alpha \neq \beta} q_{\alpha \beta}(\cdot)
\end{aligned}
$$

The machine will be able to meet the demand rate $d$ over an infinite planning horizon and reach a steady state, at a given machine age $a_{n}$, if:

$$
u_{\max } \pi_{1}^{n}(a)>d
$$

The machine age dependent limiting probability of the operational mode 1 , for a given number of failures $n$, is shown to be $\pi_{1}^{n}(a)=1 /\left(1+\frac{q_{21}(n)}{q_{12}(a)}+\frac{q_{31}}{q_{13}(a)}+\frac{q_{41}}{\omega(\cdot)}\right)$. Thus, $\pi_{1}^{n}(\cdot)$ depends on the decision variables as the transition rates are controlled through preventive maintenance. By controlling $\omega(\cdot)$, we act on the mean time before preventive maintenance. The system capacity is then described by a finite state semi-Markov chain that depends on the preventive maintenance policy. 


\section{Appendix B.}

The properties of the value function and the way to obtain the Hamilton-Jacobi-Bellman (HJB) equations can be founded in Kenné et al. (2003). Such equations describe the optimal control policies (optimality conditions) for production, repair/replacement and preventive maintenance problems. Regarding the optimality principle, we can write the HJB equations as follows:

$$
\rho v(\alpha, a, x, n)=\min _{(u, \omega) \in \Gamma(\alpha)}\left(\min _{s_{n} \in \Gamma(\alpha)}\left\{g(\cdot)+\frac{\partial v(\alpha, z, n)}{\partial a} \cdot \frac{d a(t)}{d t}+\frac{\partial v(\alpha, z, n)}{\partial x}(u(\cdot)-d)+\sum_{\beta \neq \alpha} q_{\alpha \beta}(\cdot)[v(\beta, 0, x, \psi(n)-v(\alpha, a, x, n)]\}\right)(\right.
$$

where $\beta \in \Omega$,

$$
\psi(n)= \begin{cases}0 & \text { if } \xi\left(\tau^{+}\right)=1 \text { and } \xi\left(\tau^{-}\right)=3 \\ n+1 & \text { if } \xi\left(\tau^{+}\right)=2,3 \text { and } \xi\left(\tau^{-}\right)=1 \\ n & \text { otherwise }\end{cases}
$$

$\frac{\partial v(\cdot)}{\partial x}$ and $\frac{\partial v(\cdot)}{\partial a}$ are the partial derivatives of the value function $v(\cdot)$ with respect to $x$ and to $a$; and $\tau$ denote the first jump time of $\xi(t)$.

Let us now develop the numerical methods for solving the optimality conditions given by equation (B-1) by the adaptation of Kushner's technique as in Kushner and Dupuis (1992). Let $h_{x}$ and $h_{a}$ be the finite difference interval of variables $x$ and $a$ respectively. The main idea of the approach consists in approximating $v(\alpha, a, x, n)$ by a function $v^{h}(\alpha, a, x, n)$ and the first-order partial derivatives of the value function $\frac{\partial v(\cdot)}{\partial x}$ and $\frac{\partial v(\cdot)}{\partial a}$, using the following expressions:

$$
\begin{aligned}
& \frac{\partial}{\partial x} v(\alpha, a, x, n)= \begin{cases}\frac{1}{h_{x}}\left[v^{h}\left(\alpha, a, x+h_{x}, n\right)-v^{h}(\alpha, a, x, n)\right] & \text { if } u(t)-d \geq 0 \\
\frac{1}{h_{x}}\left[v^{h}(\alpha, a, x, n)-v^{h}\left(\alpha, a, x-h_{x}, n\right)\right] & \text { otherwise }\end{cases} \\
& \frac{\partial}{\partial a} v(\alpha, a, x, n)=\frac{1}{h_{a}}\left[v^{h}\left(\alpha, a+h_{a}, x, n\right)-v^{h}(\alpha, a, x, n)\right]
\end{aligned}
$$

where $h=\left(h_{x}, h_{a}\right)$. With approximations given by equations (10) and (11) and after some manipulations, the HJB equations can be rewritten as follows:

$$
\rho v^{h}(\alpha, a, x, n)=\min _{(u, \omega) \in \Gamma(\alpha)}\left(\min _{S_{n} \in \Gamma(\alpha)}\left\{\frac{g(\cdot)}{\Upsilon_{h}^{\alpha}\left(1+\frac{\rho}{\Upsilon_{h}^{\alpha}}\right)}+\frac{1}{\left(1+\frac{\rho}{\Upsilon_{h}^{\alpha}}\right)}\left(\begin{array}{l}
P_{x}^{+} v^{h}\left(\alpha, a, x+h_{x}, n\right)+P_{x}^{-} v^{h}\left(\alpha, a, x-h_{x}, n\right)+P_{a}(\alpha) v_{h}\left(\alpha, a+h_{a}, x, n\right) \\
+\sum_{\beta \neq \alpha} P^{\beta}(\alpha) v^{h}(\alpha, a, x, n)
\end{array}\right)\right\}\right)
$$

where 
$\Upsilon_{h}^{\alpha}=\left|q_{\alpha \alpha}\right|+\frac{|u-d|}{h_{x}}, P_{x}^{+}(\alpha)=\left\{\begin{array}{l}\frac{u-d}{h_{x} \Upsilon_{h}^{\alpha}} \text { if } u-d>0 \\ 0 \quad \text { otherwise }\end{array}, P_{x}^{-}(\alpha)=\left\{\begin{array}{l}\frac{d-u}{h_{x} \Upsilon_{h}^{\alpha}} \text { if } u-d<0 \\ 0 \quad \text { otherwise }\end{array}, P_{a}(\alpha)=\frac{f(u)}{h_{a} \Upsilon_{h}^{\alpha}}, \mathrm{P}^{\beta}(\alpha)=\frac{q_{\alpha \beta}}{\Upsilon_{h}^{\alpha}}\right.\right.$

The system of equations (B-1) can be interpreted as the infinite horizon dynamic programming equation of a discrete-time, discrete-state decision process as in Boukas and Haurie (1990) and Kenné et al. (2003) for production, repair/replacement and preventive maintenance planning problems. The obtained discrete optimality conditions, described by four equations (i.e., one equation for each mode $\alpha \in \Omega=\{1,2,3,4\}$ ) can be solved using either policy improvement or successive approximation methods. In this paper, we use the policy improvement technique to obtain a solution of the approximating optimization problem. The algorithm of this technique can be found in Kushner and Dupuis (1992). 


\section{References}

Badia, F. G. and Berrade M. D.(2009). "Optimum Maintenance Policy of a periodically Inspected System under Imperfect Repair", Advances in Operations Research, vol. ??, number ??, xx-yy.

Beichelt F. (1992). "A general maintenance model and its application to repair limit replacement policies." Microelectronics and Reliability, 32(8): 1185-1196.

Boukas E.-K. and Haurie A. (1990). "Manufacturing flow control and preventive maintenance: A stochastic control approach.” IEEE Transactions on Automatic Control, 35(9): 1024-1031.

Charlot E, Kenné J.P. and Nadeau S. (2007), Optimal production, maintenance and lockout/tagout control policies in manufacturing systems, International Journal of Production Economics, 107(2), 435450.

Dellagi S., Rezg N. and Xie X. (2007). "Preventive maintenance of manufacturing systems under environmental constraints.” International Journal of Production Research, 45(5): 1233-1254.

Dehayem N.F.I., Kenné J.P. , Gharbi A. (2009),Hierarchical decision making in production and repair/replacement planning with imperfect repairs under uncertainties, European Journal of Operational Research, 198(1). 173-189

Dong-Ping, Song. (2009). "Production and preventive maintenance control in a stochastic manufacturing system , International Journal of Production Economics, 119 (1), 101-111.

F. G. Badía and M. D. Berrade (2009). "Optimum Maintenance Policy of a Periodically Inspected System under Imperfect Repair,” Advances in Operations Research, 2009, 13 pages.

Gabriella B., Rommert D. and Robin P. N. (2008). "Maintenance and Production: A Review of Planning Models." Complex System Maintenance Handbook. S. London.

Gharbi A., Kenné J. P. and Beit M. (2007). "Optimal safety stocks and preventive maintenance periods in unreliable manufacturing systems." International Journal of Production Economics, 107(2): 422-434. 
Gharbi A and Kenné J.P., Optimal Production Control Problem in Stochastic Multiple-Product Multiple-Machine Manufacturing Systems, IIE transactions, 35, 2003, 941-952.

Kijima M., H. Morimura and Suzuki Y. (1988). "Periodical replacement problem without assuming minimal repair.” European Journal of Operational Research, 37(2): 194-203.

Kenné J. P. and Boukas E. K. Hierarchical control of production and maintenance rates in manufacturing systems. Journal of Quality in Maintenance Engineering, 2003; 9(1): 66-82.

Kenné J.P., Boukas E.K. and Gharbi A. (2003), Control of production and corrective maintenance rates in a multiple-machine, multiple-product manufacturing system, Mathematical and Computer Modelling, 38(3-4), 351-365.

Kushner H. and Dupuis P. G. Numerical methods for stochastic control problems in continuous time. Springer-verlag; New York, 1992.

Leung, K.N.F. (2006) A note on 'A bivariate optimal replacement policy for a repairable system' Engineering Optimization, 38 (5): 621-625

Liao G.-L. (2007). “Optimal production correction and maintenance policy for imperfect process.” European Journal of Operational Research, 182(3): 1140-1149.

Makis V. and Jardine A. K. S. (1993). “A note on optimal replacement policy under general repair.” European Journal of Operational Research, 69(1): 75-82.

Pérès F. and Noyes D., (2003). "Evaluation of a maintenance strategy by the analysis of the rate of repair”. Quality and Reliability Engineering International, 19: 129-148.

Pérès-Ocon R. and Torres-Castro I., (2002). “A reliability semi-Markov model involving geometric processes”. Applied Stochastic Models in Business and Industry, 18: 157-170.

Sethi S. P. and Zhang Q. (1994). Hierarchical Decision Making in Stochastic Manufacturing Systems, Birkhâser. 
Sloan T., (2008). "Simultaneous determination of production and maintenance schedules using in-line equipment condition and yield information”. Naval research Logistics, 55.

Soro, Isaac W. Nourelfath Mustapha, Aït-Kadi. (2010), Daoud, "Performance evaluation of multi-state degraded systems with minimal repairs and imperfect preventive maintenance”, Reliability Engineering \& System Safety, 95( 2), 65-69.

Yao X., Xie X., Fu M. C. Marcus S. (2005). “Optimal Joint Preventive Maintenance and Production Policies.” Naval Research Logistics, 52(7): 668-681.

Yuan L. Z., Richard C. M. Yam and Zuo M. J. (2001). “Optimal replacement policy for a deteriorating production system with preventive maintenance.” International Journal of Systems Science, 32(10): 1193-1198. 